\title{
Organic matter in interplanetary dust particles
}

\author{
G. J. Flynn ${ }^{1}$, L. P. Keller ${ }^{2}$, S. Wirick ${ }^{3}$, and C. Jacobsen ${ }^{3}$ \\ ${ }^{1}$ Dept. of Physics, SUNY-Plattsburgh, 101 Broad St, Plattsburgh NY 12901 USA \\ email: flynngj@plattsburgh.edu \\ ${ }^{2}$ NASA Johnson Space Center, NASA Rt. 1, Houston TX, 77058 USA \\ ${ }^{3}$ Dept. of Physics, SUNY- Stony Brook, Stony Brook NY 11794
}

\begin{abstract}
Anhydrous interplanetary dust particles (IDPs), which are the most mineralogically primitive extraterrestrial materials available for laboratory analysis, contain several percent organic matter. The high O:C and N:C ratios suggest the organic matter in the anhydrous IDPs is significantly less altered by thermal processing than the organic matter in meteorites. X-ray Absorption Near-Edge Structure (XANES) spectroscopy and infrared spectroscopy demonstrate the presence of $\mathrm{C}=\mathrm{C}$, most likely as C-rings, $\mathrm{C}=\mathrm{O}$, and aliphatic $\mathrm{C}-\mathrm{H}_{2}$ and $\mathrm{C}^{-} \mathrm{H}_{3}$ in all the IDPs examined. A D-rich spot, containing material that is believed to have formed in a cold molecular cloud, has C-XANES and infrared spectra very similar to the organic matter in the anhydrous IDPs, possibly indicating a common formation mechanism. However the primitive organic matter in the IDPs differs from the interstellar/circumstellar organic matter characterized by astronomical infrared spectroscopy in the relative strengths of the asymmetric aliphatic $\mathrm{C}-\mathrm{H}_{2}$ and $\mathrm{C}-\mathrm{H}_{3}$ absorptions, with the IDP organic having a longer mean chain length. If both types of organic matter originated by the same process, this may indicate the interstellar organic matter has experienced more severe radiation processing than the organic matter in the primitive IDPs.
\end{abstract}

Keywords. Astrochemistry, astrobiology, infrared: general

\section{Introduction}

Interplanetary dust particles (IDPs) are small fragments from asteroids and comets that enter the Earth's atmosphere, decelerating slowly over distances of tens of kilometers, and settling towards the Earth's surface. Since the 1970's, NASA has collected IDPs from the Earth's stratosphere (Brownlee 1985), where the concentration of terrestrial dust is relatively low. These IDPs span the size range from $\sim 5 \mu \mathrm{m}$ to $>50 \mu \mathrm{m}$, though most of the particles that are collected are $\sim 10 \mu \mathrm{m}$ in diameter.

The IDPs have a similar elemental composition to that of the CI carbonaceous chondrite meteorites, which are similar to the composition of the Solar photosphere for all but the most volatile elements (Anders \& Grevesse 1989) and are believed to represent the elemental composition of the non-volatile material of the Solar Nebula. However, the IDPs contain, on average, about 12 wt-\% C (Thomas et al. 1994), which is more than three times the $\mathrm{C}$-abundance of the most C-rich meteorite, and are similarly enriched relative to CI in several moderately-volatile minor elements (Flynn et al. 1996).

Anders (1989) suggested that, since many of the IDPs decelerate so gently that they are not significantly heated, the IDPs might have contributed a significant amount of pre-biotic organic matter to the surface of the early Earth, providing important material for the origin of life. At the time of Anders paper little was known about the amount or the type(s) of organic matter in the IDPs. Because of their small size, the organic 


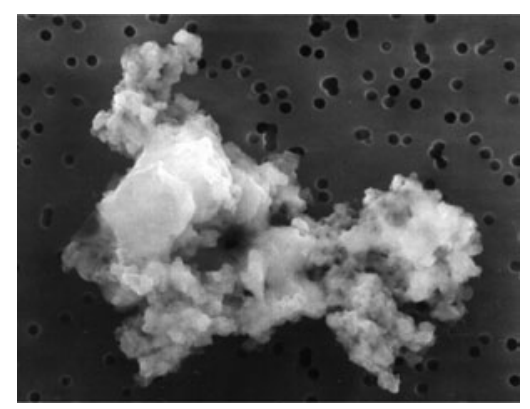

Figure 1. Scanning electron microscope image of an interplanetary dust particle (IDPs), measuring $\sim 10 \mu \mathrm{m}$ in its largest dimension, collected by NASA from the Earth's stratosphere. The texture shows the individual subunits, which are glass or discrete mineral grains, typically sub-micron in size, held together by an organic coating. (NASA photo)



Figure 2. X-ray absorption image of a portion of an ultramicrotome section of L2008R15 (top), and (bottom) a carbon map derived by comparing the absorption just below the $\mathrm{C}$ K-edge, which $\mathrm{C}$ absorbs only weakly, with the absorption just above the $\mathrm{C}$ K-edge, which $\mathrm{C}$ absorbs strongly. Bright areas have high C. (Image width $\sim 1 \mu \mathrm{m}$.)

matter in the IDPs cannot be studied using the conventional laboratory techniques that are applied to much larger samples, such as the meteorites.

We characterize the carbonaceous material in the IDPs using two synchrotron-based instruments at the National Synchrotron Light Source (NSLS) at Brookhaven National Laboratory (Upton, New York USA). A Scanning Transmission X-ray Microscope (STXM), having a spatial resolution of $\sim 35 \mathrm{~nm}$, is employed to determine the carbon abundance and map the distribution of carbon in $\sim 100 \mathrm{~nm}$ thick ultramicrotome sections of the IDPs (see Figure 2). At carbon-rich spots, X-ray Absorption Near-Edge Structure (XANES) spectroscopy is used to determine the $\mathrm{C}, \mathrm{N}$, and $\mathrm{O}$ functional groups, as described in Flynn et al. (2003). A microscope-based Fourier Transform Infrared Spectrometer ( $\mu$ FTIR) is used to identify functional groups with absorptions in the mid-IR. Combining data from the two instruments allows us to detect a variety of organic functional groups at the micron or sub-micron scale.

The majority of the IDPs are anhydrous, showing no evidence of hydrous silicates, but some contain hydrous minerals, suggesting these IDPs experienced aqueous alteration after incorporation into a parent body such as an asteroid. The anhydrous IDPs are generally fine-grained, porous aggregates, called chondritic porous (or CP) IDPs. A typical $\sim 10 \mu \mathrm{m} \mathrm{CP} \mathrm{IDP} \mathrm{contains} \sim 10^{4}$ or more individual subunits (see Figure 1 ). The 
mean bulk density of the CP IDPs is $\sim 0.6 \mathrm{gm} / \mathrm{cc}$ (Flynn \& Sutton 1991), although most of the anhydrous minerals they are made of have densities $>3 \mathrm{gm} / \mathrm{cc}$. This indicates a typical CP IDP has a very high porosity. Carbon maps of the CP IDPs show that most of the individual grains are coated with carbonaceous material, which appears to be the "glue" that holds these porous structures together (see Figure 2). The hydrous IDPs are significantly more compact, with a mean density of $\sim 1.9 \mathrm{gm} / \mathrm{cc}$ (Flynn \& Sutton 1991).

Comparison of the mineralogy of IDPs with that of other types of extraterrestrial materials, including various types of meteorites and the samples of Comet $81 \mathrm{P} / \mathrm{Wild}$ 2 collected by the Stardust spacecraft, indicates that the "CP IDPs remain the most cosmochemically primitive astromaterials" (Ishii et al. 2008). Thus, the fine-grained, porous, anhydrous IDPs are the best samples to examine in order to characterize the primitive organic matter of our Solar System.

\section{Organic Matter in Primitive, Anhydrous IDPs}

The organic matter in 8 anhydrous IDPs was characterized by C-XANES spectroscopy and $\mu$-FTIR spectroscopy. The C-XANES analyses are performed on ultramicrotome sections, typically 70 to $100 \mathrm{~nm}$ thick. The sections were prepared by embedding each particle in liquid elemental S, allowing the S to cool, then sectioning the S-bead containing the sample. The sections are deposited on TEM grids having a silicon monoxide substrate, thus avoiding exposure of the samples to carbon-bearing materials, such as embedding epoxy and substrates, during the preparation.

In general, $\mathrm{C}$ is inhomogeneously distributed, as shown in Figure 2. Different C-rich spots in the same ultramicrotome section frequently exhibit different C-XANES spectra, as shown in Figure 3. Most C-rich regions show both the $\mathrm{C}=\mathrm{C}$ absorption, at $\sim 285 \mathrm{eV}$, and the $\mathrm{C}=\mathrm{O}$ absorption feature, at $\sim 288.5 \mathrm{eV}$. The $\mathrm{C}$-rich regions also show a weaker absorption at $\sim 286.5 \mathrm{eV}$, attributed to $\mathrm{O}$ on a C-ring, C-C, or C-N bonding. Some spectra show other features, such as the aliphatic C-H* Rybergh resonances at $\sim 289 \mathrm{eV}$ (Stohr 1992). Variations in the center position and the width of a specific peak indicate differences in the bonding environment of the particular functional group. Differences in the relative areas of two peaks indicate a variation in the ratio of the two functional groups. Thus, the organic matter in these IDPs exhibits differences in the ratios of the various organic functional groups at the micron-scale, suggesting it is a mixture of several organic molecules.

The mean ratio of the area of the $\mathrm{C}=\mathrm{C}$ to $\mathrm{C}=\mathrm{O}$ absorption in the 8 anhydrous IDPs we measured is 0.92 (Flynn et al. 2003). This is quite different from the ratio of 1.29 that we find for organic matter in an ultramicrotome section of the Murchison meteorite, and suggests that the organic matter in the anhydrous IDPs contains more $\mathrm{O}$ than the organic matter in Murchison. As a direct test of this result, we can determine the C:N:O ratio in the IDP organic matter by measuring the increase in x-ray absorption at the $\mathrm{K}$-edge energies of $\mathrm{C}, \mathrm{N}$, and $\mathrm{O}$. However, determining the $\mathrm{O}$ : $\mathrm{C}$ ratio is complicated by $\mathrm{O}$ being present in both organic matter and minerals.

The N-XANES and O-XANES spectra were measured in a few of the anhydrous IDPs. Figure 4 shows the combined C-, N-. and O-XANES spectra of an anhydrous IDP, L2009D11. Because of the low N-concentration, the N-XANES spectra are generally noisy. The $\mathrm{N}$ absorption features in two of the three anhydrous IDPs measured, L2005*A3 and L2011R11, are very broad and difficult to assign to a specific functional group. The third anhydrous IDP, L2008H9, has a much stronger N-XANES spectrum. No carbon is found at the N-rich spots in L2008H9, and the N-XANES spectrum is consistent with a nitride. 




Figure 3. C-XANES spectra of seven different areas on an ultramicrotime section of L2009D11. an anhydrous IDP. Differences in the center positions and widths of the $\mathrm{C}=\mathrm{C}$ absorption $(\sim 285 \mathrm{eV})$ and the $\mathrm{C}=\mathrm{O}$ absorption $(\sim 288.5 \mathrm{eV})$ indicate differences in the nearest neighbor atoms of these functional groups at different locations while differences in the areas of the two peaks indicate different ratios of the $\mathrm{C}=\mathrm{C}$ and $\mathrm{C}=\mathrm{O}$ functional groups.

The dominant O-host in most of the anhydrous IDPs is a silicate, either olivine or pyroxene. However, $\mathrm{O}$ is also found co-located with $\mathrm{C}$. The pre-edge absorption in the OXANES spectra of these $\mathrm{C}$-rich areas is consistent with $\mathrm{C}=\mathrm{O}$, confirming our attribution of the $288.5 \mathrm{eV}$ feature in the C-XANES spectrum to $\mathrm{C}=\mathrm{O}$.

In one anhydrous IDP, L2011*B2, the organic coating was pushed away from the edge of the silicate grain during microtoming, providing an isolated area of IDP organic matter for analysis. We determined the $\mathrm{C}: \mathrm{N}: \mathrm{O}$ ratio to be $\mathrm{C}_{100} \mathrm{~N}_{10} \mathrm{O}_{50}$ for the organic matter in this C-rich area by measuring the increase in absorption at the K-edge of each of these elements and using the known x-ray absorption cross-sections. The inferred composition of $\mathrm{C}_{100} \mathrm{~N}_{10} \mathrm{O}_{50}$ has much higher O:C and $\mathrm{N}$ : $\mathrm{C}$ ratios than are reported in the acid insoluble organic matter extracted from the Murchison meteorite, which has $\mathrm{C}_{100} \mathrm{~N}_{1.8} \mathrm{O}_{12}$ (Zinner 1988).

Based on measurements by Cody, Sandford et al. (2006) have shown that the O:C and $\mathrm{N}: \mathrm{C}$ ratios of the organic matter extracted from meteorites increase with increasing primitiveness (i.e., a lesser degree of thermal metamorphism). The organic matter in L2011*B2 exhibits even higher O:C and $\mathrm{N}: \mathrm{C}$ ratios than the organic matter in the least thermally metamorphosed meteorite. This suggests the IDP organic matter has experienced even less thermal metamorphism since its formation, consistent with the 


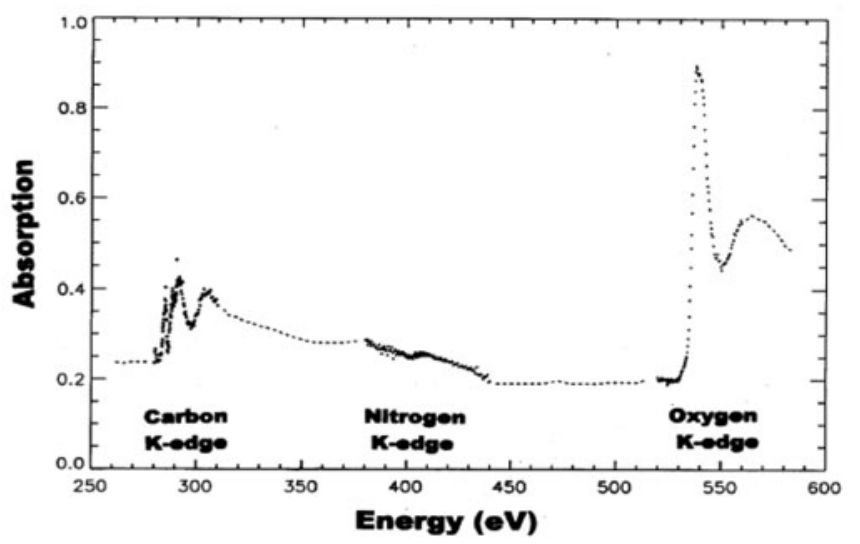

Figure 4. Combined C-, N-, and O-XANES spectra of L2009D11, an anhydrous IDP with a large D hot spot.

mineralogical observation that the anhydrous, porous IDPs are more primitive than any type of meteorite.

The high intensity of the synchrotron light allows us to perform $\mu$-FTIR analyses with a small beamspot. However, the size-scale for the infrared analysis is still limited by diffraction. We can obtain good quality spectra of the C-H stretching region, at a wavelength of $\sim 3.4 \mu \mathrm{m}$, with 3 to $5 \mu \mathrm{m}$ analysis spots. However, good quality spectra of the $\mathrm{C}=\mathrm{O}$ and $\mathrm{Si}-\mathrm{O}$ regions of the spectrum require analysis spots of 7 to $10 \mu \mathrm{m}$, comparable to the size of the whole $\sim 10 \mu \mathrm{m}$ IDP. Even for the C-rich IDPs, the infrared spectrum is typically dominated by the $\mathrm{Si}-\mathrm{O}$ absorption, which is near $10 \mu \mathrm{m}$ (see Figure 5). The aliphatic $\mathrm{C}_{-} \mathrm{H}_{2}$ and $\mathrm{C}-\mathrm{H}_{3}$ stretching absorptions, near $3.4 \mu \mathrm{m}$, have been detected in all IDPs examined thus far. However, the aromatic $\mathrm{C}-\mathrm{H}$ absorption is below the detection limit in most IDPs. The $\mathrm{C}-\mathrm{C}$ and $\mathrm{C}=\mathrm{O}$ absorption features are only detected by infrared spectroscopy in IDPs having a relatively high C-content (see Figure 5).

\section{Organic Matter in Hydrous IDPs}

The hydrous IDPs contain, on average, about the same amount of $\mathrm{C}$ as the anhydrous IDPs (Thomas et al. 1994). We characterized the organic matter in 5 hydrous IDPs. In general, the C-XANES spectra of the C-rich spots in the hydrous IDPs are quite similar to those in the anhydrous IDPs, exhibiting strong $\mathrm{C}=\mathrm{C}$ and $\mathrm{C}=\mathrm{O}$ absorptions and a weaker absorption feature at $\sim 296.5 \mathrm{eV}$. The mean $\mathrm{C}=\mathrm{C}$ to $\mathrm{C}=\mathrm{O}$ peak area ratio for the 5 hydrous IDPs is 0.96 , very similar to the 0.92 value for the 8 anhydrous IDPs. The $\mathrm{C}-\mathrm{H}$ stretching region of the infrared spectra of the hydrous IDPs is also quite similar to that of the anhydrous IDPs, These similarities in the types and abundance of organic matter in the anhydrous and the hydrous IDPs suggest that the bulk of the organic matter in the IDPs was formed prior to the aqueous alteration event, which most likely took place after incorporation of the IDPs into their parent bodies. Thus, the organic matter in the IDPs appears to have formed very early in Solar System history, or may even precede the formation of the Solar System.

\section{Non-Solar Organic Matter in the IDPs}

The isotopic ratios of most elements vary over only a narrow range for Solar System materials. Material that preserved the record of its pre-Solar history is usually identified 

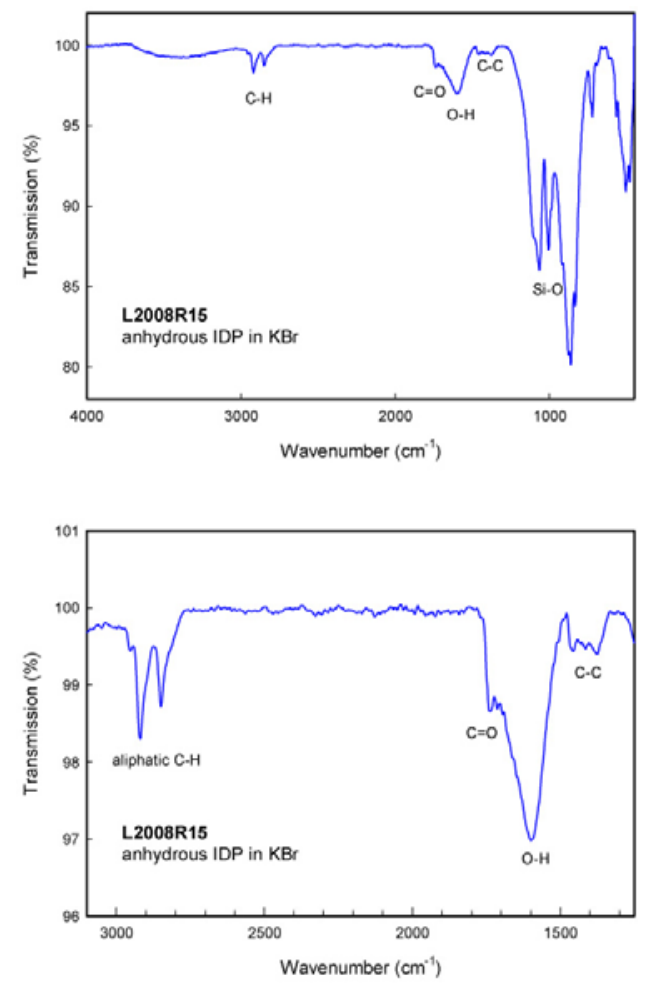

Figure 5. (top) The mid IR-spectrum of L2008R15, an anhydrous IDP, showing the organic and silicate features. (bottom) The region of this mid-IR spectrum from 1300 to $3100 \mathrm{~cm}^{-1}$, showing the organic signatures of $\mathrm{C}-\mathrm{C}, \mathrm{C}=\mathrm{O}$, and $\mathrm{C}-\mathrm{H}$ in the spectrum of L2008R15.

by isotopic ratios outside the Solar range. In the case of organic matter, isotopic ratios of $\mathrm{H}, \mathrm{C}, \mathrm{N}$, or O that are well outside the range seen in the Solar System point to a non-Solar origin for the material. Some IDPs have localized hot-spots showing extremely high D:H ratios, suggesting the D-carrier was formed in a cold molecular cloud (Messenger 2000). We analyzed the D-hot spot in a particularly D-rich IDP, L2009D11, using C-XANES. We also analyzed L2009D11 using $\mu$-FTIR (Keller et al. 2004). The D hot-spot in L2009D11 is coincident with a $\mathrm{C}$-rich region of the IDP, indicating the D-host is organic, rather than water, which is the other likely H-rich phase. The C-XANES spectrum of the D hot-spot shows the same three absorption features, at $\sim 285 \mathrm{eV}, 286.5 \mathrm{eV}$ and $288.5 \mathrm{eV}$, that we detect in the other IDPs. The $\mathrm{C}=\mathrm{C}$ and $\mathrm{C}=\mathrm{O}$ peak area ratio is 0.76 in the $\mathrm{D}$ hot-spot, lower than the mean of 0.92 for the anhydrous IDPs, but within the range of values for the 8 anhydrous IDPs. The infrared spectrum of L2009D11 has a C-H stretching region consistent with the other anhydrous IDPs, showing a strong aliphatic $\mathrm{C}_{-} \mathrm{H}_{2}$ absorption and a weaker $\mathrm{C}_{-} \mathrm{H}_{3}$ absorption feature. The aromatic $\mathrm{C}-\mathrm{H}$ feature, at $\sim 3.3 \mu \mathrm{m}$, is below our detection limit, suggesting that the D-carrier is an aliphatic hydrocarbon (Keller et al. 2004).

We can directly compare the $\mathrm{C}-\mathrm{H}$ stretching region of the infrared spectrum of the primitive organic matter in the anhydrous IDPs, including L2009D11, with astronomical spectra of interstellar/circumstellar dust. The astronomical spectra generally show roughly equal depths for the asymmetric aliphatic $\mathrm{C}-\mathrm{H}_{2}$ and $\mathrm{C}-\mathrm{H}_{3}$ absorptions (Sandford et al. 1991), while the primitive organic matter in the IDPs has a mean ratio of $\sim 2.5$ for the asymmetric aliphatic $\mathrm{C}-\mathrm{H}_{2}$ and $\mathrm{C}^{-} \mathrm{H}_{3}$ absorptions. This suggests the mean length for 
the aliphatic chains in the anhydrous IDPs is significantly longer than has been observed for the organic matter in interstellar/circumstellar sites. If both types of organic matter were produced in similar processes, the difference in aliphatic chain length might have resulted from radiation processing of the interstellar/circumstellar organic matter.

\section{Delivery of Organic Matter to the Earth by the IDPs}

The orbits of particles in the size range of the IDPs, generally 5 to $50 \mu \mathrm{m}$ in diameter, are significantly perturbed by solar radiation. The Poynting-Robertson drag force causes an $\sim 10 \mu \mathrm{m}$ IDP to spiral inwards, towards the Sun. A particle starting in the asteroid belt reaches $1 \mathrm{AU}$ in 20,000 to 100,000 years (Flynn 1989). Particles released by comets have a wider range of space exposure times. However, even IDPs produced by collisions in the Kuiper Belt spiral into the Sun on a time-scale short compared to the age of the Solar System. Thus, IDPs spend most of the life incorporated in larger bodies, most likely asteroids and comets, both of which are associated with bands or trails of dust detected in the infrared. During this time they are shielded from ionizing radiation.

However, once they are released from the parent body, the IDPs are exposed to ionizing radiation. The most damaging radiation is likely to be UV or x-rays, which can cause photo-ionization, but this radiation penetrates only a few hundred nanometers into the sample. Thus, even in an $\sim 10 \mu \mathrm{m}$ IDP, we expect the interior to be shielded from the most damaging radiation.

However, modeling by Zagorski (2007) suggests that after 100 years in space in our Solar System the radiation dose experienced by a $10 \mu \mathrm{m}$ radius silicate particle is sufficient to sterilize the interior, and that much longer exposures will alter the organic matter, eventually resulting in complete degradation. Our results demonstrate that a significant amount of organic matter survives exposure to the radiation experienced in the inner Solar System for times of $\sim 20,000$ to $\sim 100,000$ years in particles as small as $\sim 10 \mu \mathrm{m}$. Thus, the organic matter delivered to the primitive Earth by the IDPs may have been important to the origin of life on Earth.

\section{Conclusions}

Organic matter is abundant in both the anhydrous and hydrous IDPs. Both the mineralogy and the high $\mathrm{O}$ and $\mathrm{N}$ content of the organic matter in anhydrous IDPs indicates these particles contain the least processed samples of the early organic matter of our Solar System. This organic matter survived the heating experienced by IDPs during atmospheric deceleration, demonstrating that IDPs contribute a significant amount of pre-biotic organic matter to the surface of the Earth, which (Anders 1989) suggested may be important for the origin of life. Silicates and the aliphatic $\mathrm{C}-\mathrm{H}$, carbonyl $(\mathrm{C}=\mathrm{O})$, and C-ring functional groups dominate the infrared and C-XANES spectra of both the anhydrous and the hydrous IDPs, but the aromatic $\mathrm{C}-\mathrm{H}$ functional group is usually below the detection limit. The infrared and C-XANES spectra of the organic matter in both the anhydrous and the hydrous IDPs are very similar to each other, indicating that most of the pre-biotic organic matter of our Solar System formed by a process that preceded aqueous processing. The aliphatic $\mathrm{C}-\mathrm{H}_{2}$ to $\mathrm{C}-\mathrm{H}_{3}$ ratio in the IDPs is much higher than in astronomical spectra of interstellar/circumstellar dust regions, indicating the aliphatic organic matter in the IDPs occurs in longer chains and suggesting the interstellar/circumstellar organic matter may have experienced significant radiation processing. 


\section{Acknowledgements}

This work was supported by a NASA Exobiology grant. The measurements were performed on beamlines U10B and X1A of the National Synchrotron Light Source at Brookhaven National Laboratory.

\section{References}

Anders, E. 1989, Nature, 342, 255

Anders, E., \& Grevesse, N. 1989, Geochimica et Cosmochimica Acta, 53, 197

Brownlee, D. E. 1985, Ann. Rev. Earth \& Planetary Sci., 13, 147

Flynn, G. J. 1989, Icarus, 77, 287

Flynn, G. J., Bajt, S., Sutton, S. R., Zolensky, M. E., Thomas, K. L., \& Keller, L. P. 1996, in: Physics; chemistry; and dynamics of interplanetary dust, Astronomical Society of the Pacific Conference Series 310, 291ff

Flynn, G. J., Keller, L. P., Feser, M., Wirick, S., \& Jacobsen, C. 2003, Geochimica et Cosmochimica Acta, 674791

Flynn, G. J., \& Sutton, S. R. 1991, in: Cosmic dust particle densities - Evidence for two populations of stony micrometeorites, Lunar and Planetary Science Conference, 21st, Proceedings, Lunar and Planetary Institute, Houston, TX, p. 541

Ishii, H. A., Bradley, J. P., Dai, Z. R., Rong, Z., Chi, M., Kearsley, A. T., Burchell, M. J., Browning, N. D., \& Molster, F. 2008, Science, 319, 447

Keller, L. P., Messenger, S., Flynn, G. J., Clemett, S., Wirick, S., \& Jacobsen, C. 2004, Geochimica et Cosmochimica Acta, 68, 2577

Messenger, S. 2000, Nature, 404, 968

Sandford, S. A., Allamandola, L. J., Tielens, A. G., Sellgren, K., Tapia, M., \& Pendleton, Y. 1991, ApJ, 371, 607

Sandford, S. A., et al., 2006, Science, 314, 1721

Stohr, J. 1992, NEXAFS Spectroscopy, Springer-Verlag

Thomas, K. L., Keller, L. P., Blanford, G. E., \& McKay, D. S. 1994, in: Quantitative analysis of carbon in anhydrous and hydrated interplanetary dust particles, Analysis of interplanetary Dust, AIP Conf. proc. \#310, (American Institute of Physics), p. 165

Zagorski, P. A. 2007, Origin of life and Evolution of the Biosphere, 37, 351

Zinner, E. 1988, in: Interstellar cloud material in meteorites, Meteorites and the early solar system, (University of Arizona Press, Tucson, AZ), p. 956

\section{Discussion}

ZINNER: There is a subgroup of IDPs which the ${ }^{15} \mathrm{~N}$ excesses are much higher in all the other IDP, and the same IDP have also much higher abundance of presolar silicates. Have you done any analysis on these IDPs?

FLYNN: I can't tell you that we haven't because most of all the IDPs haven't been looked at for isotopes. We analyzed two IDPs that have large deuterium excesses and one IDP that has a large nitrogen excess. We have not yet found the source of the nitrogen excess but at least in one IDP we are able to analyze the source of the deuterium excess. But many of the IDPs that we've looked at but haven't been looked at the ion probe may well be in that category as well. We try to get very primitive particles.

NITTLER: We have been analyzing one of the most isotopically primitive IDPs yet identified in terms of its presolar grain abundance and isotopic composition. We have Raman spectra of the organic matter in this IDPs and it shows an extremely disordered signature quite comparable to laboratory experiments of radiation possessed organic matter. There is evidence for radiation possessing of organics in some of the primitive IDPs. 
SANDFORD: You spoke of the IDP organics as being a monolithic entity but obviously we have to keep open the possibility there's more than one population of organics there. In fact it could be this one of differences that you have additional materials in IDPs you don't see in the meteorites.

FLYNN: We see as many as five or six different distinguishable C-XANES spectra of organic matter in some of the IDPs but typically we see only two or three. Except for the largest particles because of the imprint size of the beam of the infrared we typically can't look for that but in the IDPs that are big enough, we've been able to look in the infrared, we see distinct differences from spot to spot in the $\mathrm{CH}_{3}$ to $\mathrm{CH}_{2}$ ratios as well. The organic matter in the IDPs is not homogenous at the scale that we are able to measure at and we look at the capability of measuring at even finer scale.

ZiURYs: Your signatures of the $\mathrm{C}=\mathrm{C}$, you said they were probably aromatic. Is that really $\mathrm{C}=\mathrm{C}$ ? Could it be $\mathrm{C}$ triple bond $\mathrm{C}$ ?

FLynN: They are $\mathrm{C}=\mathrm{C}$, but whether it is $\mathrm{C}=\mathrm{C}$ and a ring is the ambiguity.

ZIURYS: Your sort of organic content looks more like gas phase in a interstellar molecule. You see lots of carbon groups and a lot of carbon chains.

Alexander: I take a slightly different spin on your infrared spectrum. The Murchison has undergone a lot of aqueous alteration. We already know that affect the aliphatic materials in the IOM. We also know it removes a lot of soluble aliphatic materials, as does the isolation of organic materials when you make your IOM residues. I would suggest the reason you are seeing difference in infrared spectra with your IDPs is that they haven't undergone the aqueous alteration that removes the aliphatic component, the soluble aliphatic component, nor they undergone the isolation process that most IOMs have undergone as well.

FLYNN: But the most hydrous IDPs that we analyzed have a spectrum that virtually identical to most anhydrous IDPs that we analyzed.

Alexander: You may not have a sink for removing the water etc and soluble organic materials.

BernsteIn: With regards to the elevated $\mathrm{CH}_{2}$ to $\mathrm{CH}_{3}$ ratio in IDPs relative to meteorites, although that is consistent with longer chain length, it is also consistent with aliphatic rings. So it's definitely not a dead lock for straight chain molecules. Moreover, it's simply like that having aromatic compound with some of the rings reduced which is also consistent with radiation processing proposed by Greg Sloan. 


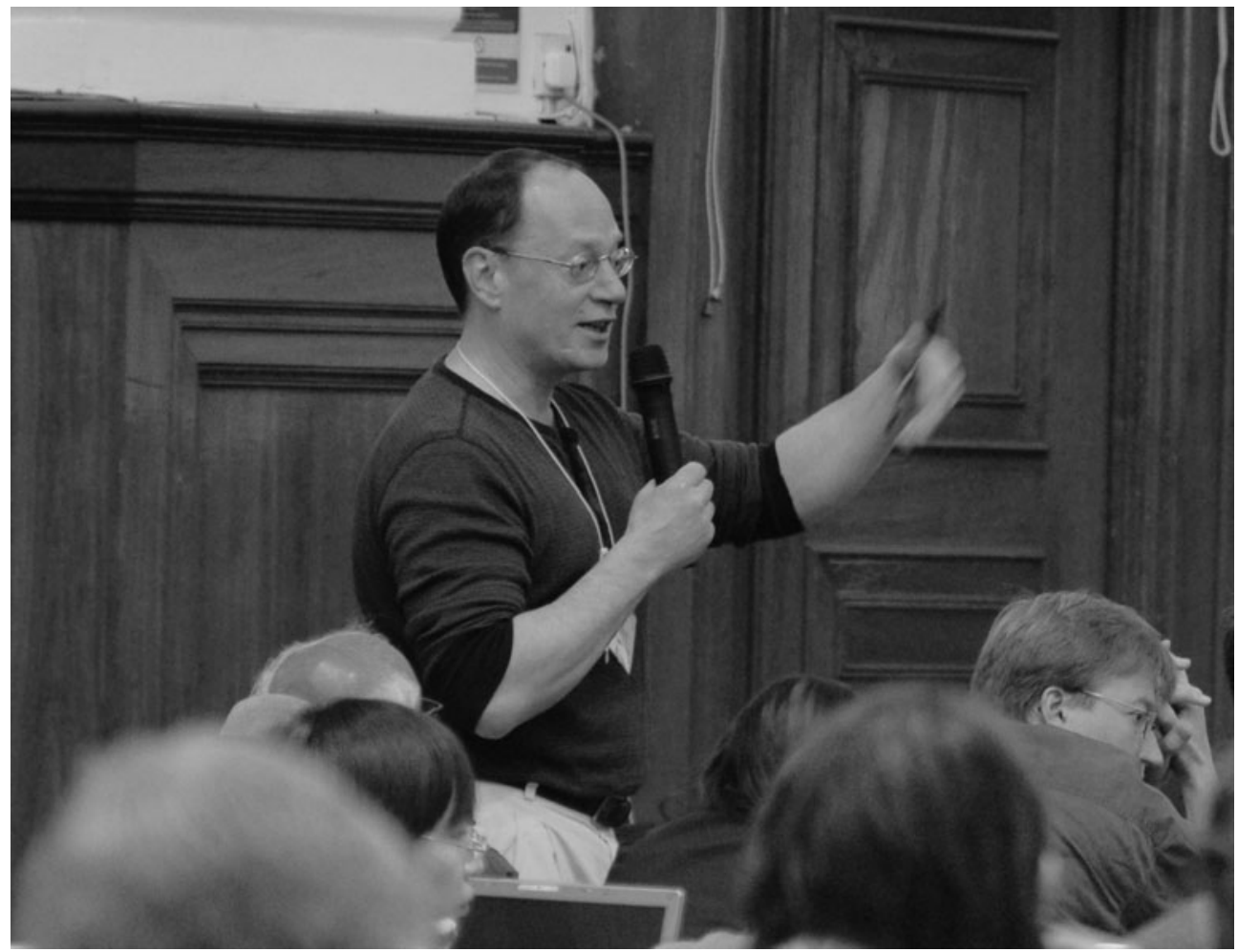

George Cody arguing a point of view (photo by Sze-Leung Cheung). 